\title{
Paramagnetism-based Refinement Strategy for the Solution Structure of Human $\alpha$-Parvalbumin
}

\author{
Irfan Baig, ${ }^{1}$ Ivano Bertini, ${ }^{* 1}$ Cristina Del Bianco, ${ }^{1}$ Yogesh Kumar Gupta, ${ }^{1}$ \\ Yong-Min Lee, ${ }^{1}$ Claudio Luchinat, ${ }^{2}$ and Alessandro Quattrone ${ }^{3}$
}

${ }^{1}$ Magnetic Resonance Centre and Department of Chemistry, University of Florence, Via Luigi Sacconi, 6, 50019, Sesto Fiorentino, Italy ${ }^{2}$ Magnetic Resonance Centre and Department of Agricultural Biotechnology, P.le delle Cascine 28, 50144, Florence, Italy ${ }^{3}$ Medical Genetics Unit, IRCCS CSS, Viale Padre Pio, 71013, San Giovanni Rotondo (FG), Italy.

\section{Supplementary Material}

Table S1. Acquisition parameters for NMR experiments performed on Human parvalbumin.

Table S2. ${ }^{1} \mathrm{H}$ and ${ }^{15} \mathrm{~N}$ Resonance assignments for Human Parvalbumin

Table S3. ${ }^{13} \mathrm{C}$ Resonance assignments for Human Parvalbumin

Table S4. Stereospecific assignment for diastereotopic pairs in Parvalbumin

Table S5. $\phi$ torsion angle restraints of Parvalbumin

Table S6. $\Psi$ torsion angle restraints of Parvalbumin

Table S7. The PCS and RDC values used in solution structure calculations for $\mathrm{Dy}^{3+}$ substituted Parvalbumin. 
Table S1. Acquisition parameters for NMR experiments performed on Human parvalbumin.

\begin{tabular}{|c|c|c|c|c|c|c|}
\hline \multirow[t]{3}{*}{ Experiments } & \multicolumn{3}{|c|}{ Dimension of acquired data } & \multicolumn{3}{|c|}{ Spectral width } \\
\hline & \multicolumn{3}{|c|}{ (Nucleus) } & \multicolumn{3}{|c|}{$(\mathbf{H z})$} \\
\hline & $\mathbf{t}_{1}$ & $\mathbf{t}_{2}$ & $\mathbf{t}_{3}$ & $\mathbf{F}_{1}$ & $\mathbf{F}_{2}$ & $\overline{F_{3}}$ \\
\hline${ }^{1} \mathrm{H}-{ }^{15} \mathrm{~N}-\mathrm{HSQC}$ & $256\left({ }^{15} \mathrm{~N}\right)$ & $2048\left({ }^{1} \mathrm{H}\right)$ & & 2500 & 11261 & \\
\hline$\left[{ }^{1} \mathrm{H}-{ }^{1} \mathrm{H}\right]-\mathrm{NOESY}$ & $\left.1024{ }^{1} \mathrm{H}\right)$ & $2048\left({ }^{1} \mathrm{H}\right)$ & & 9756 & 9756 & \\
\hline$\left[{ }^{1} \mathrm{H}-{ }^{1} \mathrm{H}\right]-\mathrm{TOCSY}$ & $1024\left({ }^{1} \mathrm{H}\right)$ & $1976\left({ }^{1} \mathrm{H}\right)$ & & 9756 & 9756 & \\
\hline$\left[{ }^{1} \mathrm{H}-{ }^{13} \mathrm{C}\right]-\mathrm{TROSY}-\mathrm{HNCO}$ & $128\left({ }^{13} \mathrm{C}\right)$ & $2048\left({ }^{1} \mathrm{H}\right)$ & & 2012 & 8012 & \\
\hline $\mathrm{CBCA}(\mathrm{CO}) \mathrm{NH}$ & $128\left({ }^{13} \mathrm{C}\right)$ & $64\left({ }^{15} \mathrm{~N}\right)$ & $1024\left({ }^{1} \mathrm{H}\right)$ & 13227 & 2413 & 8865 \\
\hline CBCANH & $128\left({ }^{13} \mathrm{C}\right)$ & $64\left({ }^{15} \mathrm{~N}\right)$ & $1024\left({ }^{1} \mathrm{H}\right)$ & 13227 & 2413 & 8865 \\
\hline $\mathrm{CC}(\mathrm{CO}) \mathrm{NH}$ & $96\left({ }^{13} \mathrm{C}\right)$ & $32\left({ }^{15} \mathrm{~N}\right)$ & $1024\left({ }^{1} \mathrm{H}\right)$ & 13192 & 7257 & 11261 \\
\hline${ }^{13} \mathrm{C}(\mathrm{H}) \mathrm{CCH}-\mathrm{TOCSY}$ & $256\left({ }^{13} \mathrm{C}\right)$ & $100\left({ }^{13} \mathrm{C}\right)$ & $1024\left({ }^{1} \mathrm{H}\right)$ & 15625 & 15625 & 9124 \\
\hline${ }^{1} \mathrm{H}-{ }^{13} \mathrm{C}-\mathrm{HSQC}$ & $256\left({ }^{13} \mathrm{C}\right)$ & $1024\left({ }^{1} \mathrm{H}\right)$ & & 8865 & 14963 & \\
\hline $\mathrm{HNCO}$ & $96\left({ }^{13} \mathrm{C}\right)$ & $64\left({ }^{15} \mathrm{~N}\right)$ & $1024\left({ }^{1} \mathrm{H}\right)$ & 2474 & 2413 & 8865 \\
\hline HNHA & $256\left({ }^{1} \mathrm{H}\right)$ & $40\left({ }^{15} \mathrm{~N}\right)$ & $1024\left({ }^{1} \mathrm{H}\right)$ & 7575 & 2431 & 7575 \\
\hline HNHB & $136\left({ }^{1} \mathrm{H}\right)$ & $40\left({ }^{15} \mathrm{~N}\right)$ & $1024\left({ }^{1} \mathrm{H}\right)$ & 8339 & 2500 & 8389 \\
\hline${ }^{1} \mathrm{H}-{ }^{15} \mathrm{~N}-\mathrm{HSQC}-\mathrm{NOESY}$ & $126\left({ }^{1} \mathrm{H}\right)$ & $48\left({ }^{15} \mathrm{~N}\right)$ & $1024\left({ }^{1} \mathrm{H}\right)$ & 9765 & 2403 & 9765 \\
\hline${ }^{1} \mathrm{H}-{ }^{13} \mathrm{C}-\mathrm{HSQC}-\mathrm{NOESY}$ & $224\left({ }^{1} \mathrm{H}\right)$ & $80\left({ }^{13} \mathrm{C}\right)$ & $1024\left({ }^{1} \mathrm{H}\right)$ & 9765 & 13157 & 9765 \\
\hline${ }^{1} \mathrm{H}-{ }^{15} \mathrm{~N}-\mathrm{HSQC}^{\mathrm{b}}(\mathrm{PCS})$ & $256\left({ }^{15} \mathrm{~N}\right)$ & $2048\left({ }^{1} \mathrm{H}\right)$ & & 2500 & 11261 & \\
\hline${ }^{1} \mathrm{H}-{ }^{15} \mathrm{~N}-\mathrm{HSQC}\left(J_{N H}\right.$-split $)$ & $256\left({ }^{15} \mathrm{~N}\right)$ & $2048\left({ }^{1} \mathrm{H}\right)$ & & 2500 & 11261 & \\
\hline
\end{tabular}


Table S2. ${ }^{1} \mathrm{H}$ and ${ }^{15} \mathrm{~N}$ Resonance assignments for Human Parvalbumin.

\begin{tabular}{|c|c|c|c|c|c|c|c|c|c|c|c|c|}
\hline Residue & $\mathbf{N}$ & $\mathrm{HN}$ & HA & & HB2 & HB3 & HG2 & HG3 & HD2 & HD3 & HE2 & HE3 \\
\hline $\mathrm{M} 1$ & 122.51 & 8.26 & 4.53 & & QB 1.99 & & 2.50 & 2.57 & & & & \\
\hline $\mathrm{s} 2$ & 118.54 & 8.81 & 4.76 & & QB 3.80 & & & & & & & \\
\hline M3 & 125.16 & 9.20 & 4.36 & & QB 2.14 & & QG 2.56 & & & & & \\
\hline$\overline{T 4}$ & 105.84 & 7.72 & 4.67 & & HB 4.58 & & QG2 1.27 & & & & & \\
\hline D 5 & 119.34 & 7.76 & 4.38 & & QB 2.83 & & & & & & & \\
\hline L 6 & 118.54 & 7.40 & 4.41 & & 1.76 & 1.35 & HG 1.12 & & QD1 0.85 & & & \\
\hline L7 & 117.75 & 7.99 & 4.70 & & QB 1.59 & & HG 1.17 & & QD1 0.89 & & & \\
\hline N8 & 117.48 & 8.49 & 4.43 & & QB 2.85 & & & & $\begin{array}{r}7.72 \\
\end{array}$ & 7.13 & & \\
\hline A 9 & 128.87 & 9.28 & 3.94 & & QB 1.54 & & & & & & & \\
\hline E 10 & 117.22 & 8.63 & 4.07 & & QB 2.05 & & QG 2.30 & & & & & \\
\hline D 11 & 121.19 & 7.56 & 4.43 & & 2.91 & 2.89 & & & & & & \\
\hline 112 & 119.34 & 8.08 & 3.61 & & HB 1.92 & & QG2 1.15 & HG12 1.92 & HG13 0.88 & QD1 0.91 & & \\
\hline K 13 & 118.28 & 7.90 & 3.94 & & 1.87 & 1.72 & 1.55 & \begin{tabular}{|r|}
1.32 \\
\end{tabular} & QD 0.96 & & QE 2.96 & \\
\hline K 14 & 118.81 & 7.83 & 3.96 & & 1.88 & 1.79 & 1.33 & 1.80 & QD 1.56 & & QE 2.96 & \\
\hline A 15 & 121.72 & 8.35 & 4.24 & & QB 1.28 & & & & & & & \\
\hline $\mathrm{V} 16$ & 112.19 & 8.81 & 3.97 & & HB 2.29 & & QG1 1.06 & QG2 1.23 & & & & \\
\hline G 17 & 106.63 & 7.86 & 3.81 & 3.89 & & & & & & & & \\
\hline A 18 & 121.72 & 7.36 & 3.98 & & QB 0.87 & & & & & & & \\
\hline F19 & 115.63 & 7.30 & 5.16 & & 3.50 & 2.60 & & & QD 6.716 & & QE 6.44 & \\
\hline$S 20$ & 116.42 & 7.53 & 4.16 & & QB 3.94 & & & & & & & \\
\hline A 21 & 122.78 & 8.23 & 4.40 & & QB 1.37 & & & & & & & \\
\hline T22 & 119.34 & 8.62 & 4.42 & & HB 3.97 & & QG2 1.32 & & & & & \\
\hline D 23 & 120.39 & 8.22 & 4.43 & & QB 2.89 & & & & & & & \\
\hline$S 24$ & 111.66 & 7.67 & 4.40 & & QB 4.23 & & & & & & & \\
\hline F 25 & 123.84 & 9.56 & 3.57 & & QB 2.66 & & & & QD 6.71 & & QE 6.42 & \\
\hline D 26 & 109.81 & 8.34 & 4.45 & & 2.51 & & & & & & & \\
\hline $\mathrm{H} 27$ & 122.25 & 8.35 & 4.43 & & 1.88 & 1.59 & & & & & & \\
\hline K 28 & 120.92 & 6.89 & 4.01 & & QB 1.62 & & 1.14 & 0.74 & QD 1.75 & & QE 3.10 & \\
\hline K 29 & 117.22 & 6.97 & 4.35 & & 1.99 & 1.78 & QG 0.94 & & QD 1.3 & & QE 2.95 & \\
\hline $\mathrm{F} 30$ & 118.28 & 8.18 & 4.01 & & QB 2.49 & & & & QD 6.76 & & & \\
\hline$\overline{F 31}$ & 116.16 & 8.66 & 4.28 & & 3.38 & 2.95 & & & QD 7.35 & & QE 6.95 & \\
\hline Q 32 & 118.01 & 7.81 & 3.97 & & QB 2.23 & & QG 2.48 & & & & 7.77 & 6.67 \\
\hline M 33 & 119.07 & 8.64 & 3.91 & & QB 1.97 & & 2.87 & 2.43 & & & & \\
\hline V34 & 114.57 & 8.24 & 3.74 & & HB 1.33 & & QG1 0.87 & $\begin{array}{|ll|}\text { QG2 } & 0.61\end{array}$ & & & & \\
\hline G 35 & 104.78 & 7.15 & 3.91 & 4.28 & & & & & & & & \\
\hline L 36 & 119.34 & 7.28 & 3.97 & & 1.66 & & HG 1.59 & & QD2 1.43 & & & \\
\hline K 37 & 115.36 & 8.05 & 3.89 & & 1.87 & 1.66 & QG 1.37 & & QD 1.67 & & QE 2.95 & \\
\hline K 38 & 114.04 & 7.02 & 4.33 & & 1.99 & 1.70 & QG 1.36 & & QD 1.67 & & QE 2.83 & \\
\hline K 39 & 118.01 & 6.99 & 3.99 & & 2.01 & 2.17 & QG 1.744 & & QD 1.136 & & QE 2.52 & \\
\hline$S 40$ & 117.48 & 9.24 & 4.35 & & QB 4.07 & & & & & & & \\
\hline A 41 & 123.31 & 8.83 & 4.05 & & QB 1.43 & & & & & & & \\
\hline D 42 & 115.89 & 8.28 & 4.32 & & QB 2.53 & & & & & & & \\
\hline
\end{tabular}




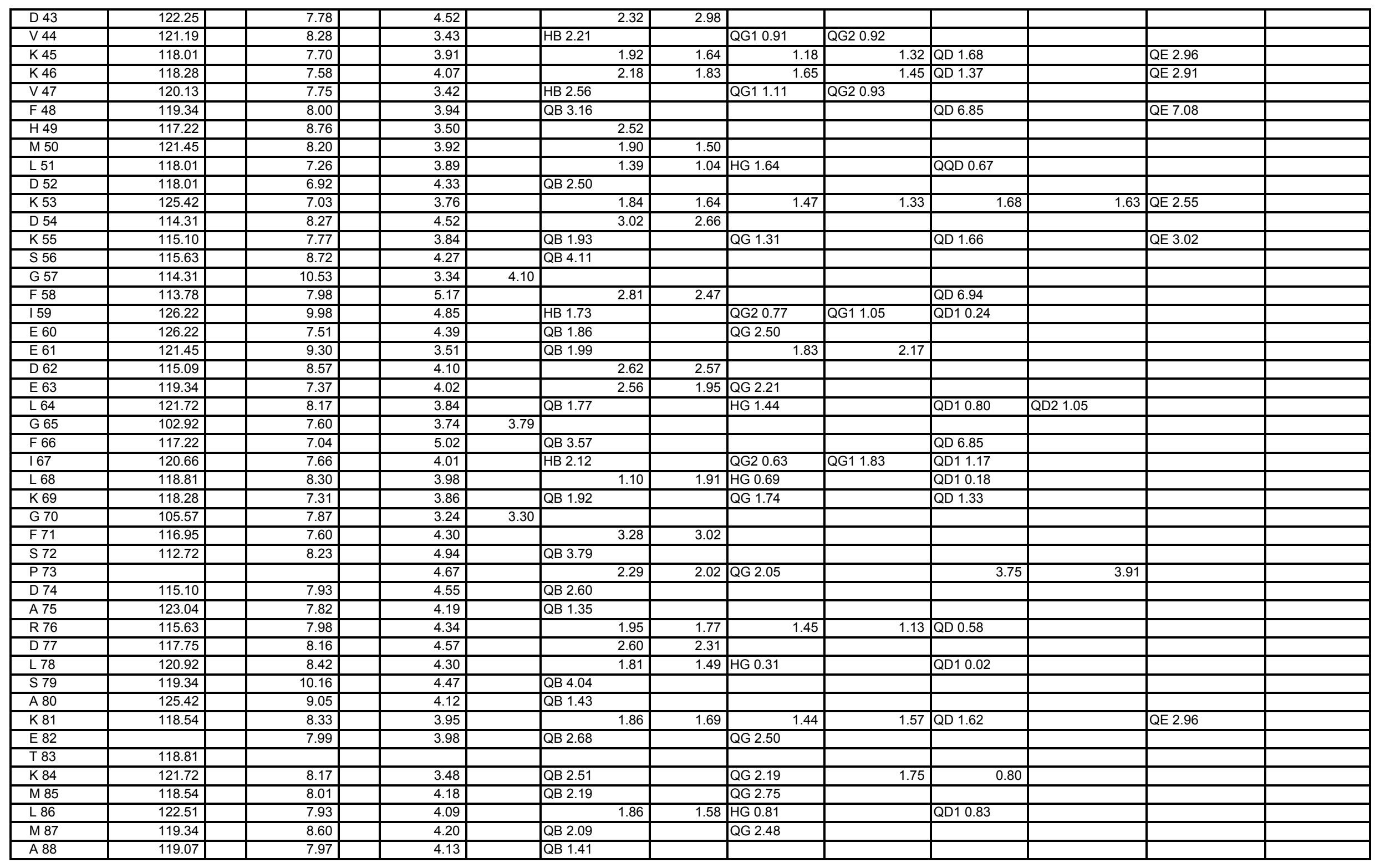




\begin{tabular}{|c|c|c|c|c|c|c|c|c|c|c|c|}
\hline A 89 & 117.48 & 7.47 & 4.15 & & QB 1.37 & & & & & & \\
\hline G 90 & 101.60 & 8.16 & 3.24 & 4.12 & & & & & & & \\
\hline D 91 & 120.66 & 8.73 & 4.71 & & 2.83 & 2.36 & & & & & \\
\hline K 92 & 127.01 & 8.27 & 4.18 & & 1.91 & 1.46 & QG 1.40 & & QD 1.64 & & QE 2.72 \\
\hline D 93 & 115.63 & 8.42 & 4.62 & & QB 3.03 & & & & & & \\
\hline G 94 & 108.48 & 7.55 & 3.76 & 3.85 & & & & & & & \\
\hline D 95 & 120.66 & 8.34 & 4.44 & & 2.81 & 2.40 & & & & & \\
\hline G96 & 112.19 & 10.12 & 3.46 & 3.94 & & & & & & & \\
\hline K 97 & 115.89 & 7.79 & 4.70 & & 1.61 & 1.47 & 1.02 & 0.87 & 0.83 & 1.02 & QE 1.92 \\
\hline 198 & 123.31 & 8.70 & 5.10 & & HB 2.04 & & QG2 1.18 & HG12 1.68, HG13 & 30.94 & QD1 0.82 & \\
\hline$\overline{G 99}$ & 116.95 & 9.78 & 4.04 & 4.73 & & & & & & & \\
\hline V100 & 120.92 & 8.29 & 2.15 & & HB 1.16 & & QG1 0.36 & QG2 0.59 & & & \\
\hline D 101 & 121.72 & 8.00 & 4.41 & & 2.67 & 2.71 & & & & & \\
\hline E102 & 121.98 & 7.68 & 4.06 & & QB 2.58 & & QG 2.33 & & & & \\
\hline F103 & 122.25 & 8.90 & 3.98 & & QB 3.09 & & & & & 6.16 & \\
\hline S 104 & 114.04 & 8.21 & 3.84 & & QB 4.42 & & & & & & \\
\hline T105 & 119.87 & 8.10 & 3.89 & & HB 4.12 & & QG2 1.18 & & QD 6.16 & & QE 6.83 \\
\hline L 106 & 122.25 & 7.87 & 3.89 & & 1.66 & & HG 1.5 & & & QD1 0.59 & \\
\hline V 107 & 117.22 & 7.42 & 3.00 & & HB 1.63 & & QG1 - 0.41 & QG2 0.42 & & & \\
\hline A 108 & 121.19 & 7.89 & 4.09 & & QB 1.46 & & & & & & \\
\hline E 109 & 115.63 & 7.94 & 3.93 & & 2.26 & 2.07 & 2.51 & 2.31 & & & \\
\hline S 110 & 122.78 & 7.62 & 4.16 & & QB 4.14 & & & & & & \\
\hline
\end{tabular}


Table S3. ${ }^{13} \mathrm{C}$ Resonance assignments for Human Parvalbumin

\begin{tabular}{|c|c|c|c|c|c|}
\hline Residue & CA & CB & CG & $C D$ & CE \\
\hline M 1 & 53.03 & 31.53 & 29.21 & & \\
\hline S2 & 54.09 & 62.58 & & & \\
\hline M 3 & 54.90 & 28.60 & 29.96 & & \\
\hline T4 & 60.52 & 65.95 & CG2 19.28 & & \\
\hline D 5 & 53.06 & 39.44 & & & \\
\hline L 6 & 53.23 & 42.48 & 23.38 & CD1 21.65 & \\
\hline L7 & 49.57 & 41.96 & 24.39 & CD1 19.84 & \\
\hline N 8 & 50.28 & 37.58 & & & \\
\hline A 9 & 53.16 & 16.15 & & & \\
\hline E 10 & 56.56 & 26.14 & 33.64 & & \\
\hline D 11 & 54.47 & 36.97 & CG1 27.03 & $\begin{array}{ll}\text { CD1 } 11.51 \\
\end{array}$ & \\
\hline 112 & 63.81 & 35.77 & CG2 15.59 & & \\
\hline $\mathrm{K} 13$ & 56.84 & 29.92 & 22.31 & 26.47 & 39.30 \\
\hline $\mathrm{K} 14$ & 56.85 & 29.90 & 22.06 & 24.27 & 39.33 \\
\hline A 15 & 52.37 & 17.24 & & & \\
\hline \multirow[t]{2}{*}{$\mathrm{V} 16$} & 63.71 & 28.99 & CG1 19.54 & & \\
\hline & & & CG2 19.60 & & \\
\hline G 17 & 43.53 & & & & \\
\hline A 18 & 50.71 & 14.99 & & & \\
\hline F 19 & 53.63 & 36.16 & & & \\
\hline S 20 & 58.43 & 60.56 & & & \\
\hline A 21 & 49.07 & 16.08 & & & \\
\hline T 22 & 61.99 & 66.05 & CG2 19.43 & & \\
\hline D 23 & 55.09 & 35.03 & & & \\
\hline S 24 & 56.52 & 61.77 & & & \\
\hline$\overline{F 25}$ & 58.76 & 36.47 & & & \\
\hline D 26 & 49.14 & 39.93 & & & \\
\hline $\mathrm{H} 27$ & 56.54 & 26.31 & & & \\
\hline $\mathrm{K} 28$ & 56.77 & 28.61 & 22.38 & 26.63 & 39.25 \\
\hline K 29 & 54.08 & 29.38 & & & \\
\hline $\mathrm{F} 30$ & 60.09 & 36.99 & & & \\
\hline F 31 & 56.43 & 34.17 & & & \\
\hline Q 32 & 56.50 & 25.60 & 30.59 & & \\
\hline M 33 & 56.80 & 32.30 & 29.87 & & \\
\hline \multirow[t]{2}{*}{ V 34} & 59.81 & 29.08 & CG1 20.92 & & \\
\hline & & & CG2 18.33 & & \\
\hline G 35 & 43.41 & & & & \\
\hline \multirow[t]{2}{*}{ L 36} & 54.80 & 39.36 & 25.90 & CD1 23.15 & \\
\hline & & & & CD2 22.04 & \\
\hline K 37 & 54.75 & 26.56 & 21.40 & 29.95 & 39.41 \\
\hline K 38 & 53.05 & 30.05 & 22.16 & 26.38 & 39.37 \\
\hline K 39 & 50.33 & 29.62 & 21.93 & 24.17 & 39.71 \\
\hline S 40 & 54.16 & 63.40 & & & \\
\hline A 41 & 52.73 & 15.25 & & & \\
\hline D 42 & 54.80 & 37.92 & & & \\
\hline D 43 & 54.78 & 37.98 & & & \\
\hline \multirow[t]{2}{*}{ V 44} & 65.21 & 28.75 & CG1 21.01 & & \\
\hline & & & CG2 19.41 & & \\
\hline K 45 & 56.69 & 28.53 & 22.37 & 26.56 & 39.16 \\
\hline K 46 & 57.64 & 29.69 & 22.25 & 25.62 & 39.42 \\
\hline \multirow[t]{2}{*}{ V 47 } & 64.29 & 28.46 & CG1 20.61 & & \\
\hline & & & CG2 18.71 & & \\
\hline $\mathrm{F} 48$ & 59.40 & 35.08 & & & \\
\hline \multicolumn{6}{|l|}{$\mathrm{H} 49$} \\
\hline \multicolumn{6}{|l|}{ M 50} \\
\hline L 51 & 52.72 & 39.01 & 23.38 & 19.40 & \\
\hline D 52 & 48.72 & 35.45 & & & \\
\hline K53 & 56.84 & 29.32 & 22.13 & 26.19 & 39.39 \\
\hline D 54 & 50.13 & 36.77 & & & \\
\hline K 55 & 54.41 & 25.78 & 21.95 & 28.26 & 39.33 \\
\hline$S 56$ & 56.53 & 61.88 & & & \\
\hline
\end{tabular}




\begin{tabular}{|c|c|c|c|c|c|}
\hline G 57 & 41.82 & & & & \\
\hline F 58 & 53.15 & 41.21 & 23.78 & & \\
\hline 159 & 57.48 & 35.62 & 15.54 & CD1 11.55 & \\
\hline E 60 & 54.82 & 26.55 & 29.99 & & \\
\hline E61 & 58.42 & 27.15 & 33.85 & & \\
\hline D 62 & 53.23 & 36.19 & & & \\
\hline E 63 & 54.97 & 28.21 & 33.60 & & \\
\hline $\mathrm{L} 64$ & 54.47 & 39.29 & 21.96 & CD1 25.75 & \\
\hline G 65 & 43.86 & & & & \\
\hline F 66 & 52.82 & 36.17 & & & \\
\hline \multirow[t]{2}{*}{167} & 63.44 & 34.88 & CG1 26.72 & 10.68 & \\
\hline & & & CG2 13.78 & & \\
\hline L 68 & 55.74 & 38.62 & 22.20 & CD1 20.20 & \\
\hline $\mathrm{K} 69$ & 54.26 & 39.07 & 21.99 & 39.44 & \\
\hline G 70 & 43.22 & & & & \\
\hline F 71 & 57.54 & 36.64 & & & \\
\hline$S 72$ & 62.60 & 52.72 & & & \\
\hline $\mathrm{P} 73$ & 62.40 & 29.42 & 24.34 & 48.40 & \\
\hline D 74 & 50.39 & 37.87 & & & \\
\hline A 75 & 48.98 & 19.06 & & & \\
\hline R 76 & 51.93 & 27.97 & 24.10 & 40.86 & \\
\hline D 77 & 51.68 & 37.90 & & & \\
\hline \multirow[t]{2}{*}{$L 78$} & 51.97 & 39.13 & 23.15 & CD1 18.65 & \\
\hline & & & & CD2 24.07 & \\
\hline S 79 & 54.21 & 63.33 & & & \\
\hline A 80 & 52.85 & 15.00 & & & \\
\hline K 81 & 56.75 & 29.70 & 21.52 & 25.75 & 39.36 \\
\hline E 82 & 56.15 & 29.03 & 35.26 & & \\
\hline \multicolumn{6}{|l|}{ T 83} \\
\hline \multicolumn{6}{|l|}{ K 84} \\
\hline M 85 & 55.88 & 29.59 & 27.15 & & \\
\hline L 86 & 54.82 & 39.05 & 24.01 & CD1 21.68 & \\
\hline M 87 & 53.95 & 27.30 & 33.73 & & \\
\hline A 88 & 51.60 & 15.09 & & & \\
\hline A 89 & 50.67 & 16.27 & & & \\
\hline G 90 & 43.15 & & & & \\
\hline \multicolumn{6}{|l|}{ D 91} \\
\hline K 92 & 54.65 & 29.83 & 21.37 & 26.40 & 39.37 \\
\hline D 93 & 49.76 & 37.05 & & & \\
\hline G 94 & 44.78 & & & & \\
\hline D 95 & 50.30 & 37.40 & & & \\
\hline G 96 & 43.41 & & & & \\
\hline K 97 & 51.54 & 38.76 & 20.39 & 26.79 & 33.57 \\
\hline \multirow[t]{2}{*}{198} & 57.00 & 36.81 & CG1 25.38 & CD1 10.48 & \\
\hline & & & CG2 15.16 & & \\
\hline G 99 & 41.78 & & & & \\
\hline \multirow[t]{2}{*}{$\vee 100$} & 62.82 & 28.07 & CG1 19.09 & & \\
\hline & & & CG2 16.43 & & \\
\hline D 101 & 54.17 & 36.82 & & & \\
\hline E 102 & 55.86 & 27.07 & 34.07 & & \\
\hline F 103 & 59.61 & 37.53 & & & \\
\hline \multicolumn{6}{|l|}{ S 104} \\
\hline T105 & 63.98 & 65.96 & & & \\
\hline L 106 & 54.95 & 39.91 & 23.71 & 21.72 & \\
\hline \multirow[t]{2}{*}{ V 107} & 63.24 & 28.73 & CG1 19.02 & & \\
\hline & & & CG2 18.90 & & \\
\hline A 108 & 53.07 & 16.20 & & & \\
\hline E 109 & 53.96 & 26.81 & 33.85 & & \\
\hline$S 110$ & 58.78 & 61.88 & & & \\
\hline
\end{tabular}


Table S4. Stereospecific assignment for diastereotopic pairs in Parvalbumin

\begin{tabular}{|l|l|}
\hline $\mathrm{H} \alpha 1 / \mathrm{H} \alpha 2$ & G 35, G 90, G70, G 96 \\
\hline $\mathrm{H} \beta 2 / \mathrm{H} \beta 3$ & K 37, L 68, P 73, R 76, L 78 \\
\hline $\mathrm{H} \gamma 2 / \mathrm{H} \gamma 3$ & K 84, K 97 \\
\hline $\mathrm{CH}_{3} \gamma 1 / \mathrm{CH}_{3} \gamma 2$ & V 34, V 107 \\
\hline $\mathrm{H} \gamma 12 / \mathrm{H} \gamma 13$ & $\mathrm{I} 98$ \\
\hline $\mathrm{H} \delta 2 / \mathrm{H} \delta 3$ & P 73 \\
\hline
\end{tabular}


Table S5. $\phi$ torsion angle restraints of Parvalbumin

\begin{tabular}{|c|c|c|c|c|c|c|c|}
\hline 9 ALA PHI & -70.0 & 42 ASP- PHI & -70.0 & -30 & 98 ILE PHI & -155.0 & -85 \\
\hline 10 GLU- PHI & -70.0 & 43 ASP-PHI & -70.0 & -30 & 99 GLY PHI & -155.0 & -85 \\
\hline 11 ASP-PHI & $\begin{array}{ll}-70.0 & -30 \\
\end{array}$ & 44 VAL PHI & -70.0 & -30 & $100 \mathrm{VAL} \mathrm{PHI}$ & -70.0 & -30 \\
\hline 12 ILE PHI & $\begin{array}{ll}-70.0 & -30 \\
\end{array}$ & $45 \mathrm{LYS}+\mathrm{PHI}$ & -70.0 & -30 & 102 GLU- PHI & -80.0 & -20 \\
\hline $13 \mathrm{LYS}+\mathrm{PHI}$ & -70.0 & 46 LYS+ PHI & -70.0 & -30 & 103 PHE PHI & -70.0 & -30 \\
\hline $14 \mathrm{LYS}+\mathrm{PHI}$ & -70.0 & $47 \mathrm{VAL} \mathrm{PHI}$ & -70.0 & -30 & 104 SER PHI & -70.0 & -30 \\
\hline $15 \mathrm{ALA}$ PHI & -70.0 & 48 PHE PHI & -70.0 & -30 & 106 LEU PHI & -70.0 & -30 \\
\hline $16 \mathrm{VAL} \mathrm{PHI}$ & -70.0 & $49 \mathrm{HIS}+\mathrm{PHI}$ & -70.0 & -30 & $107 \mathrm{VAL} \mathrm{PHI}$ & -70.0 & -30 \\
\hline 17 GLY PHI & -70.0 & 50 MET PHI & -70.0 & -30 & 108 ALA PHI & -70.0 & -30 \\
\hline $27 \mathrm{HIS}+\mathrm{PHI}$ & -70.0 & 51 LEU PHI & -70.0 & -30 & 109 GLU- PHI & -70.0 & -30 \\
\hline $28 \mathrm{LYS}+\mathrm{PHI}$ & -70.0 & 58 PHE PHI & -155.0 & -85 & 69 LYS+ PHI & -70.0 & -30 \\
\hline 29 LYS + PHI & -80.0 & 59 ILE PHI & -155.0 & -85 & 70 GLY PHI & -70.0 & -30 \\
\hline 30 PHE PHI & -70.0 & 61 GLU- PHI & -70.0 & -30 & $71 \mathrm{PHE} \mathrm{PHI}$ & -70.0 & -30 \\
\hline $31 \mathrm{PHE} \mathrm{PHI}$ & -70.0 & 62 ASP-PHI & -80.0 & -20 & 80 ALA PHI & -70.0 & -30 \\
\hline 32 GLN PHI & -70.0 & 63 GLU- PHI & -80.0 & -20 & $81 \mathrm{LYS}+\mathrm{PHI}$ & -70.0 & -30 \\
\hline 33 MET PHI & -70.0 & 64 LEU PHI & -70.0 & -30 & 82 GLU- PHI & -70.0 & -30 \\
\hline $34 \mathrm{VAL}$ PHI & -70.0 & 65 GLY PHI & -70.0 & -30 & 83 THR PHI & -70.0 & -30 \\
\hline 36 LEU PHI & -70.0 & 67 ILE PHI & -80.0 & -20 & $84 \mathrm{LYS}+\mathrm{PHI}$ & -70.0 & -30 \\
\hline $37 \mathrm{LYS}+\mathrm{PHI}$ & -70.0 & 68 LEU PHI & -70.0 & -30 & 86 LEU PHI & -70.0 & -30 \\
\hline $38 \mathrm{LYS}+\mathrm{PHI}$ & $-70.0-30$ & 89 ALA PHI & -70.0 & -30 & $87 \mathrm{MET}$ PHI & -70.0 & -30 \\
\hline 41 ALA PHI & -70.0 & 90 GLY PHI & -70.0 & -30 & 88 ALA PHI & -70.0 & -30 \\
\hline
\end{tabular}

Table S6. $\psi$ torsion angle constraints of Parvalbumin

\begin{tabular}{|c|c|c|c|c|c|c|c|c|}
\hline 9 ALA PSI & -60.0 & -20 & 44 VAL PSI & -60.0 & -20 & 82 GLU- PSI & -60.0 & -20 \\
\hline 10 GLU- PSI & -60.0 & -20 & 45 LYS+ PSI & -60.0 & -20 & 83 THR PSI & -60.0 & -20 \\
\hline 11 ASP-PSI & -60.0 & -20 & 46 LYS+ PSI & -60.0 & -20 & 84 LYS+ PSI & -60.0 & -20 \\
\hline 12 ILE PSI & -60.0 & -20 & 47 VAL PSI & -60.0 & -20 & 85 MET PSI & -60.0 & -20 \\
\hline 13 LYS+ PSI & -60.0 & -20 & 48 PHE PSI & -60.0 & -20 & 86 LEU PSI & -60.0 & -20 \\
\hline $14 \mathrm{LYS}+\mathrm{PSI}$ & -60.0 & -20 & $49 \mathrm{HIS}+\mathrm{PSI}$ & -60.0 & -20 & 87 MET PSI & -60.0 & -20 \\
\hline 15 ALA PSI & -60.0 & -20 & 50 MET PSI & -60.0 & -20 & 88 ALA PSI & -60.0 & -20 \\
\hline 16 VAL PSI & -60.0 & -20 & 51 LEU PSI & -60.0 & -20 & 89 ALA PSI & -60.0 & -20 \\
\hline 17 GLY PSI & -60.0 & -20 & 58 PHE PSI & 60.0 & 175 & 90 GLY PSI & -60.0 & -20 \\
\hline $27 \mathrm{HIS}+\mathrm{PSI}$ & -60.0 & -20 & 59 ILE PSI & 60.0 & 175 & 98 ILE PSI & 50.0 & 185 \\
\hline $28 \mathrm{LYS}+\mathrm{PSI}$ & -60.0 & -20 & 61 GLU-PSI & -60.0 & -20 & 99 GLY PSI & 60.0 & 175 \\
\hline 29 LYS+ PSI & -60.0 & -20 & 62 ASP-PSI & -60.0 & -20 & 100 VAL PSI & -60.0 & -20 \\
\hline 30 PHE PSI & -60.0 & -20 & 63 GLU- PSI & -60.0 & -20 & 101 ASP-PSI & -60.0 & -20 \\
\hline $31 \mathrm{PHE}$ PSI & -60.0 & -20 & 64 LEU PSI & -60.0 & -20 & 102 GLU- PSI & -70.0 & -10 \\
\hline 32 GLN PSI & -60.0 & -20 & 65 GLY PSI & -60.0 & -20 & 103 PHE PSI & -60.0 & -20 \\
\hline 33 MET PSI & -60.0 & -20 & 67 ILE PSI & -60.0 & -20 & 104 SER PSI & -70.0 & -10 \\
\hline 34 VAL PSI & -60.0 & -20 & 68 LEU PSI & -60.0 & -20 & 105 THR PSI & -60.0 & -20 \\
\hline 36 LEU PSI & -60.0 & -20 & 69 LYS+ PSI & -60.0 & -20 & 106 LEU PSI & -60.0 & -20 \\
\hline 37 LYS+ PSI & -60.0 & -20 & 70 GLY PSI & -60.0 & -20 & 107 VAL PSI & -60.0 & -20 \\
\hline 38 LYS+ PSI & -60.0 & -20 & $71 \mathrm{PHE}$ PSI & -60.0 & -20 & 108 ALA PSI & -60.0 & -20 \\
\hline 42 ASP-PSI & -60.0 & -20 & 80 ALA PSI & -60.0 & -20 & 109 GLU- PSI & -60.0 & -20 \\
\hline 43 ASP-PSI & -60.0 & -20 & $81 \mathrm{LYS}+\mathrm{PSI}$ & -60.0 & -20 & & & \\
\hline
\end{tabular}


Table S7. The PCS and RDC values used in solution structure calculations for $\mathrm{Dy}^{3+}{ }^{-}$-substituted Parvalbumin.

\begin{tabular}{|c|c|c|c|c|c|c|c|}
\hline \multirow{2}{*}{ Residue } & \multicolumn{2}{|c|}{ PCS (ppm) } & \multirow{2}{*}{$\frac{\mathrm{RDC}(\mathrm{Hz})}{\mathrm{N}-\mathrm{H}}$} & \multirow{2}{*}{ Residue } & \multicolumn{2}{|c|}{ PCS (ppm) } & \multirow{2}{*}{$\frac{\mathrm{RDC}(\mathrm{Hz})}{\mathrm{N}-\mathrm{H}}$} \\
\hline & $\mathrm{H}_{\mathrm{N}}$ & $\mathrm{N}$ & & & $\mathrm{H}_{\mathrm{N}}$ & $\mathrm{N}$ & \\
\hline M 1 & 0.41 & 0.34 & 8.03 & S 56 & & & \\
\hline S 2 & 0.30 & 0.32 & 14.28 & G 57 & -3.64 & -3.82 & -9.33 \\
\hline M 3 & -0.26 & -0.10 & 5.91 & F 58 & & & \\
\hline T 4 & 0.09 & 0.03 & -12.40 & 159 & & & \\
\hline D 5 & 0.26 & 0.20 & -2.64 & $\mathrm{E} 60$ & & & \\
\hline L 6 & 0.34 & 0.26 & 0.83 & E 61 & -3.73 & -3.22 & \\
\hline L 7 & 0.06 & 0.16 & -17.34 & D 62 & & & \\
\hline N 8 & 0.02 & 0.03 & 6.52 & E 63 & & & \\
\hline A 9 & 0.04 & 0.01 & 21.10 & L 64 & & & \\
\hline E10 & 0.00 & 0.00 & 12.92 & G 65 & & & \\
\hline D 11 & 0.00 & -0.02 & 16.48 & F 66 & & & \\
\hline । 12 & -0.10 & -0.15 & & 167 & -1.49 & -1.64 & 4.07 \\
\hline K 13 & -0.17 & -0.30 & & L 68 & -1.87 & -1.75 & \\
\hline K 14 & -0.18 & -0.27 & 16.46 & K 69 & -1.04 & -1.32 & 22.05 \\
\hline A 15 & -0.26 & -0.33 & 16.34 & G 70 & -1.38 & -1.21 & 14.12 \\
\hline V 16 & -0.41 & -0.46 & 21.86 & F 71 & -0.85 & -0.67 & 20.56 \\
\hline G 17 & -0.42 & -0.44 & 19.77 & S 72 & -0.69 & -0.54 & 15.18 \\
\hline A 18 & -0.42 & -0.47 & 17.14 & P 73 & & & \\
\hline F 19 & -0.56 & -0.51 & 18.18 & D 74 & -0.38 & -0.37 & -17.02 \\
\hline S 20 & -0.55 & -0.48 & & A 75 & -0.72 & -0.78 & 3.51 \\
\hline A 21 & -0.51 & -0.61 & -3.87 & R 76 & -0.80 & -0.78 & -1.28 \\
\hline Т 22 & -0.11 & -0.18 & 0.51 & D 77 & & & \\
\hline D 23 & & & & L 78 & -0.85 & -1.10 & 9.49 \\
\hline S 24 & & & & S 79 & & & \\
\hline $\mathrm{F} 25$ & & & & A 80 & & & \\
\hline D 26 & & & & K 81 & & & \\
\hline H 27 & & & & E 82 & 0.29 & 0.21 & -18.66 \\
\hline K 28 & -0.67 & -0.60 & 19.61 & T 83 & & & \\
\hline K 29 & -0.42 & -0.37 & 22.97 & K 84 & & & \\
\hline $\mathrm{F} 30$ & -0.57 & -0.53 & 18.22 & M 85 & & & \\
\hline F 31 & & & & L 86 & & & \\
\hline Q 32 & & & & M 87 & & & \\
\hline M 33 & -0.13 & -0.10 & & A 88 & & & \\
\hline V 34 & -0.12 & -0.17 & 16.91 & A 89 & & & \\
\hline G 35 & -0.10 & -0.12 & 15.51 & G 90 & & & \\
\hline L 36 & 0.08 & 0.09 & 10.31 & D 91 & & & \\
\hline K 37 & 0.19 & 0.30 & 18.19 & K 92 & & & \\
\hline K 38 & 0.38 & 0.24 & 6.37 & D 93 & & & \\
\hline K 39 & 0.65 & 0.70 & 12.71 & G 94 & & & \\
\hline S 40 & 0.74 & 0.79 & 2.52 & D 95 & & & \\
\hline A 41 & 1.48 & 1.49 & -6.51 & G 96 & & & \\
\hline D 42 & 1.95 & 2.16 & 3.23 & K 97 & & & \\
\hline D 43 & 1.57 & 1.62 & -6.96 & 198 & & & \\
\hline V 44 & 1.97 & 2.12 & -13.07 & G 99 & & & \\
\hline $\mathrm{K} 45$ & 3.11 & 3.27 & -1.12 & $V 100$ & & & \\
\hline K 46 & 2.32 & 2.40 & 5.65 & D 101 & & & \\
\hline$\vee 47$ & & & & E 102 & & & \\
\hline F 48 & & & & F 103 & & & \\
\hline H 49 & & & & S 104 & & & \\
\hline M 50 & & & & $\mathrm{~T} 105$ & & & \\
\hline L 51 & & & & L 106 & & & \\
\hline D 52 & & & & V 107 & & & \\
\hline K 53 & & & & A 108 & & & \\
\hline D 54 & & & & E 109 & -1.83 & -1.59 & -9.87 \\
\hline K 55 & & & & S 110 & & & \\
\hline
\end{tabular}

